\title{
Pictograma de fadiga durante o uso de quimioterápico no tratamento do câncer de mama
}

Fatigue pictogram during chemotherapy use in breast cancer treatment

Pictograma de fatiga durante el uso de quimioterapia en el tratamiento del cáncer de mama

\section{Daniele Copello Vaz', Viviane Reis Fontes da Silva ${ }^{\text {II }}$, Roberto Carlos Lyra da Silva ${ }^{\text {III }}$, Luiz Carlos Santiago $^{\mathrm{IV}}$, Carlos Roberto Lyra da Silva ${ }^{\mathrm{V}}$}

\begin{abstract}
Resumo: Objetivo: identificar o período mais crítico da fadiga durante a quimioterapia com protocolo doxorrubicina + ciclofosfamida seguido de docetaxel em mulheres com câncer de mama utilizando o pictograma de fadiga. Método: estudo descritivo, prospectivo com abordagem quantitativa. Para um melhor monitoramento, as pacientes foram avaliadas no dia da infusão do quimioterápico e nas duas semanas seguintes por meio do contato telefônico. Resultados: na primeira etapa do tratamento, a fadiga ocorreu em menor gravidade e teve pouca interferência no desempenho das atividades diárias das mulheres. Durante a infusão do docetaxel a fadiga foi mais acentuada e recorrente, apresentando menores índices de recuperação e tendo forte impacto sobre a execução das atividades habituais. Conclusão: o pictograma de fadiga demonstrou ser um instrumento de fácil compreensão e rápida aplicação, podendo contribuir no cuidado de enfermagem na medida em que se apresenta como um importante instrumento de acompanhamento.
\end{abstract}

Descritores: Enfermagem; Fadiga; Neoplasias da mama; Antineoplásicos

Abstract: Objective: To identify the most critical period of fatigue during doxorubicin + cyclophosphamide protocol followed by docetaxel in women with breast cancer using the fatigue pictogram. Method: descriptive, prospective study with quantitative approach. For better monitoring, patients were evaluated on the day of chemotherapy infusion and the next two weeks by telephone contact. Results: In the first stage of treatment,

\footnotetext{
${ }^{\mathrm{I}}$ Enfermeira. Mestre em saúde e tecnologia no espaço hospitalar - mestrado profissional. Instituto Nacional do Câncer. Rio de Janeiro (RJ), Brasil. E-mail: dani_copello@hotmail.com. ORCID: 0000-0002-2536-2492

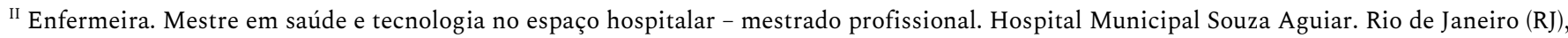
Brasil. E-mail: vivianereisfontes@gmail.com. ORCID: 0000-0003-4174-8544

III Enfermeiro. Doutor em Enfermagem. Coordenador do Programa de Pós-Graduação em Enfermagem e Biociências da Escola de Enfermagem Alfredo Pinto, Universidade Federal do Estado do Rio de Janeiro - UNIRIO. Rio de Janeiro (RJ), Brasil. E-mail: proflyra@gmail.com. ORCID: 0000-0001-9416-9525

IV Enfermeiro. Pó-Doutor em Enfermagem. Docente Permanente do Programa de Pós-Graduação em Enfermagem e Biociências da Escola de Enfermagem Alfredo Pinto. Universidade Federal do Estado do Rio de Janeiro - UNIRIO. Rio de Janeiro (RJ), Brasil. E-mail: luisolitrio@gmail.com. ORCID: 0000-0002-9725-4626

v Enfermeiro. Pós-Doutor em Enfermagem. Docente Permanente dos Programas de Pós-Graduação em Enfermagem e Enfermagem e Biociências da Escola de Enfermagem Alfredo Pinto. Universidade Federal do Estado do Rio de Janeiro - UNIRIO. Rio de Janeiro (RJ), Brasil. Email: profunirio@gmail.com. ORCID: 0000-0002-4327-6272
} 
fatigue was less severe and had little interference with women's daily activities. During docetaxel infusion, fatigue was more pronounced and recurrent, presenting lower recovery rates and having a strong impact on the performance of usual activities. Conclusion: The fatigue pictogram proved to be an easy to understand and quick application instrument, and can contribute to nursing care as it is an important monitoring tool.

Descriptors: Nursing; Fatigue; Breast neoplasms; Antineoplastics

Resumen: Objetivo: identificar el período más crítico de fatiga durante el protocolo de doxorrubicina + ciclofosfamida seguido de docetaxel en mujeres con cáncer de mama utilizando el pictograma de fatiga. Método: estudio descriptivo, prospectivo, con enfoque cuantitativo. Para una mejor monitorización, los pacientes fueron evaluados el día de la infusión de quimioterapia y las siguientes de las semanas por contacto telefónico. Resultados: En la primera etapa del tratamiento, la fatiga fue menos severa y tuvo poca interferencia con las actividades diarias de las mujeres. Durante la infusión de docetaxel, la fatiga fue más pronunciada y recurrente, presentando tasas de recuperación más bajas y teniendo un fuerte impacto en el desempeño de las actividades habituales. Conclusión: El pictograma de fatiga demostró ser un instrumento fácil de entender y de aplicación rápida, y puede contribuir al cuidado de enfermería, ya que es una herramienta de monitoreo importante.

Descriptores: Enfermería; Fatiga Neoplasias de mama; Antineoplásicos

\section{Introdução}

Apesar dos avanços na detecção precoce e tratamento, o câncer continua sendo uma das doenças mais temidas, devido à sua associação não só com a morte, mas também com a diminuição da qualidade de vida. Entre os sintomas mais comuns relacionados ao câncer e seu tratamento estão a dor, depressão e fadiga. ${ }^{1}$

Pesquisa realizada principalmente em mulheres com câncer de mama sugere que a prevalência da fadiga moderada a grave durante a quimioterapia pode chegar a $60 \% .^{2}$ Este sintoma pode estar presente durante o processo estressante do diagnóstico, podendo aumentar durante a conduta terapêutica e normalmente decai com o término do tratamento. No entanto, pode persistir em pacientes clinicamente curados. ${ }^{3}$

Embora seja considerado um dos sintomas mais prevalentes e debilitantes, nem sempre é valorizado pelos profissionais da área da saúde. A fadiga pode ser definida como uma sensação desagradável e multifatorial, pois envolve aspectos físicos, psíquicos e emocionais, varia em relação à duração e intensidade e está relacionada a um cansaço que não diminui com estratégias de restauração de energia, prejudicando assim a execução de atividades rotineiras. ${ }^{4}$ 
3 | Vaz DC, Silva VRF, Silva RCL, Santiago LC, Silva CRL

Vários fatores podem contribuir para o manejo inadequado deste sintoma, tais como: entendimento incompleto e imperfeito da fisiologia da fadiga, limitação eficaz do seu tratamento e o desconhecimento de ferramentas para sua avaliação. Por outro lado, muitas vezes, os pacientes acabam não relatando sua condição por alguns motivos, dentre eles, por considerarem o sintoma normal visto a gravidade do seu estado geral ou ainda, por não acreditarem na capacidade dos médicos em oferecer intervenções benéficas ou por desconhecerem a existência de tratamentos efetivos. ${ }^{5}$

Desta forma, o desafio é chamar ainda mais a atenção dos profissionais de saúde para a importância do reconhecimento e abordagem ativa da fadiga relacionada ao câncer e seu tratamento. A subjetividade e a natureza multidimensional são fatores que dificultam a avaliação da fadiga.

A estratégia comumente utilizada para tentar diminuir o viés na mensuração dos sintomas subjetivos em pacientes com câncer, mas que necessita de avaliação regular e comparativa é a utilização de escala visual analógica ou escala numérica. Nesse sentido, merecem destaque os instrumentos para identificação e acompanhamento clínico dos sintomas subjetivos, tais como, dor, depressão e fadiga. ${ }^{1}$

Diante do exposto, é razoável admitir que esta propositura encontra sua relevância no cotidiano de profissionais que acompanham pacientes acometidos pela fadiga durante e após a quimioterapia de controle do câncer de mama, uma vez que torna possível avaliar a evolução de mulheres que convivem com essa condição. Portanto, a questão de pesquisa foi: qual o período mais crítico da fadiga experimentado por mulheres com câncer de mama durante a quimioterapia com protocolo doxorrubicina + ciclofosfamida seguido de docetaxel? Para responder este questionamento, o estudo teve como objetivo identificar o período mais crítico da fadiga durante a quimioterapia com protocolo doxorrubicina + ciclofosfamida seguido de docetaxel em mulheres com câncer de mama utilizando o pictograma de fadiga. 
Pictograma de fadiga durante o uso de quimioterápico no tratamento do câncer de mama $\mid 4$

\section{Método}

Trata-se de um estudo descritivo, prospectivo e com abordagem quantitativa, desenvolvido na central de quimioterapia de uma clínica particular, localizada na cidade do Rio de Janeiro, Brasil. O período de coleta de dados foi compreendido entre os meses de março a dezembro de 2017, cuja a amostra foi constituída por conveniência, resultando em 15 (quinze) mulheres com idade igual ou superior a 18 anos, todas com câncer de mama, independente do estágio da doença, iniciando tratamento quimioterápico com doxorrubicina + ciclofosfamida seguido de docetaxel, neo-adjuvante ou adjuvante. As participantes da pesquisa assinaram o Termo de Consentimento Livre e Esclarecido, após serem informadas quanto aos objetivos.

Os critérios de inclusão consistiram em: ter idade igual ou superior a 18 anos; estar ciente do diagnóstico de câncer e do tratamento; ser capaz de comunicar-se verbalmente para responder aos questionamentos durante a coleta de dados e ter acesso à uma linha telefônica para ser acompanhada à distância. Os critérios de exclusão foram pacientes que tiveram a necessidade da mudança do esquema terapêutico devido à resistência aos antineoplásicos durante o tratamento ou que apresentaram alguma toxicidade grave às drogas e óbito antes do término do tratamento.

Para estabelecer o vínculo entre profissional e paciente, a pesquisadora foi responsável pela realização da primeira consulta de enfermagem. Ao término da consulta e após o esclarecimento de todas as dúvidas, a paciente foi abordada para participar da pesquisa. Nesse momento, lhe fora apresentado e explicado o instrumento para avaliação da fadiga. Cabe destacar que o protocolo quimioterápico ao qual essas mulheres foram submetidas é composto por quatro aplicações de doxorrubicina + ciclofosfamida, seguidas por quatro aplicações de docetaxel, todas com intervalos de 21 dias e duração média de cinco meses. As respostas sobre o pictograma de fadiga, foram colhidas antes do início e ao final do tratamento, momentos em que a pesquisadora reforçou como 
5 | Vaz DC, Silva VRF, Silva RCL, Santiago LC, Silva CRL

interpretar o pictograma de fadiga, fornecendo a escala de avaliação impressa, colada num papel cartão e plastificada, que serviu para as próximas etapas do acompanhamento à distância, via telefone.

Para um melhor monitoramento da fadiga ao longo de todo o tratamento quimioterápico, a pesquisadora foi responsável pela avaliação da fadiga em todos os ciclos da quimioterapia ambulatorial e entrou em contato com a paciente por via telefônica no oitavo e décimo quinto dia após cada aplicação, tendo em vista que, de acordo com a literatura médica, os sintomas são mais prevalentes nestes períodos da quimioterapia e por meio do pictograma fornecido na primeira consulta, foi capaz de avaliar melhor o nível da fadiga em seu domicílio. Sendo assim, cada mulher foi avaliada em relação ao nível de fadiga em oito consultas presenciais e dezesseis contatos telefônicos, totalizando 24 momentos. Os dados obtidos foram tabulados e analisados por meio do software Microsoft Excel Office, versão 2010, apresentados em tabelas e gráficos nas quais foram calculadas as frequências dos achados e discutidos de acordo com a literatura vigente.

O Pictograma de Fadiga é um instrumento de composto por duas perguntas que avaliam a intensidade da fadiga e o seu impacto na realização de atividades diárias. Cada item é avaliado por meio de ilustrações legendadas. Para cada pergunta têm-se como resposta cinco desenhos legendados. Por meio dessa escala é possível avaliar a intensidade da fadiga (nada cansado, um pouquinho cansado, moderadamente cansado, muito cansado e extremamente cansado) e o impacto da fadiga nas atividades diárias (eu consigo fazer tudo que habitualmente faço, eu consigo fazer quase tudo que habitualmente faço, eu consigo fazer algumas das coisas que habitualmente faço, eu só faço o que tenho que fazer e eu consigo fazer muito pouco), ${ }^{4}$ conforme ilustrado na Figura 1. 
Pictograma de fadiga durante o uso de quimioterápico no tratamento do câncer de mama 6

Figura 1 - Pictograma de fadiga.

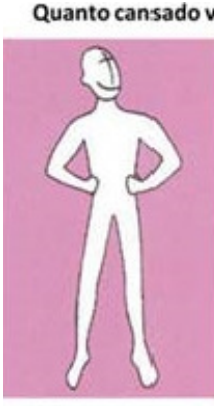

Nada cansado

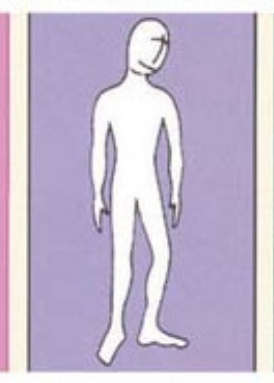

Um pouquinho cansado

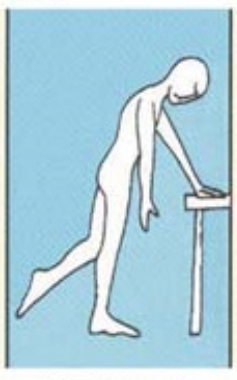

Moderadamente cansado

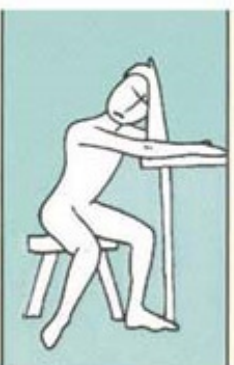

Muito cansado

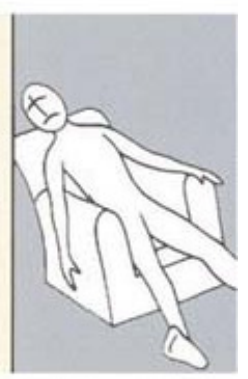

Extremamente cansado

Quanto a sensação de cansaço te impede de fazer o que você quer fazer?
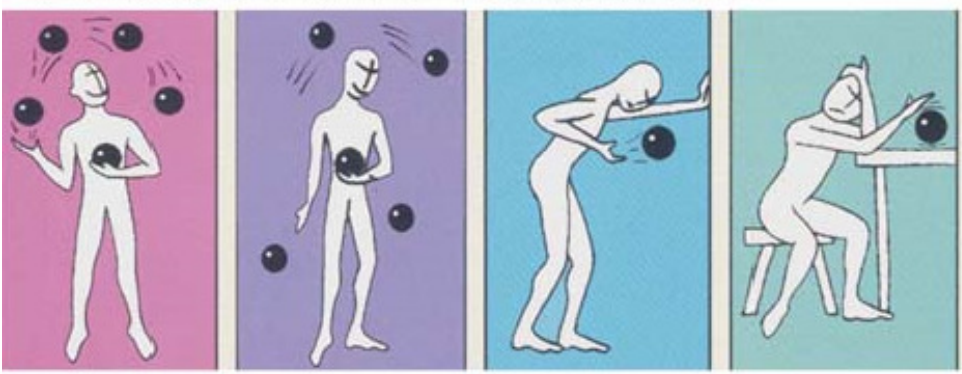

Eu só faço o que tenho que fazer

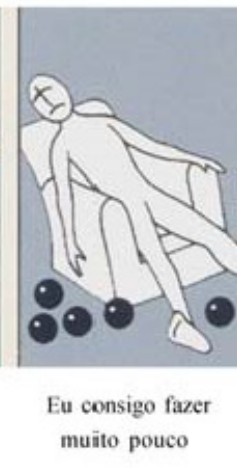

Eu consigo fazer quase Eu consigo fazer algumas tudo que habitualmente das coisas que habitualmente faço que habitualmente faço faço

Fonte: Pictograma de Fadiga: uma alternativa para avaliação da intensidade e impacto da fadiga, 2009. Brasil. ${ }^{5}$

Este instrumento foi validado no Brasil, ${ }^{5}$ tendo como participantes do estudo, pacientes com câncer em diversos estágios, cuidadores dos pacientes com câncer e estudantes de enfermagem. Os resultados revelaram que as propriedades psicométricas do Pictograma da Fadiga foram satisfatórias apenas para os doentes e não para os acompanhantes e estudantes. Esse instrumento demonstrou ter boa validade e boa estabilidade em pacientes com câncer em diversos estágios da doença. ${ }^{6}$

Este estudo respeitou as diretrizes sobre pesquisa com seres humanos, norteadas pela resolução 466/12 do Conselho Nacional de Saúde. Obteve aprovação pelo Comitê de Ética da Universidade Federal do Estado do Rio de Janeiro em 30/06/2014, recebendo parecer favorável sob o número 704.968. 


\section{Resultados}

Participaram do estudo 15 mulheres com câncer de mama submetidas ao tratamento quimioterápico com o protocolo doxorrubicina + ciclofosfamida seguido de docetaxel. Uma paciente foi excluída, pois apresentou toxicidade ao tratamento a partir da segunda aplicação do docetaxel, sendo necessário a mudança do protocolo.

As participantes tinham idade média de 57,3 anos. Em relação ao estado civil, a maioria era casada $35,71 \%(n=5)$, seguida por solteira $28,57 \%(n=4)$, separada $21,43 \%(n=3)$ e viúva $14,29 \%$ $(\mathrm{n}=2)$. Quanto ao nível de escolaridade, $50 \%(\mathrm{n}=7)$ tinham o nível médio, 21,43\% ( $\mathrm{n}=3)$ o fundamental, 14,29\% $(\mathrm{n}=2)$ superior completo, $7,14 \%(\mathrm{n}=1)$ o ensino primário e apenas $7,14 \%$ (n=1) possui pós-graduação.

Em termos de ocupação, a maior parte era aposentada $42,86 \%(n=6)$, seguida por assalariada $35,71 \%(\mathrm{n}=5)$, do lar $14,29 \%(\mathrm{n}=2)$ e autônoma $7,14 \%(\mathrm{n}=1)$. No que se refere ao histórico da doença atual, havia um predomínio da modalidade neo-adjuvante $57,14 \%(\mathrm{n}=8)$. As demais mulheres fizeram o tratamento adjuvante $42,86 \%(n=6)$, em que todas realizaram esvaziamento axilar, $66,67 \% \quad(\mathrm{n}=4)$ foram submetidas a mastectomia, $33,33 \% \quad(\mathrm{n}=2) \quad$ a segmentectomia e apenas $16,66 \%$ ( $n=1)$ fez a reconstrução da mama.

A metade da amostra apresentou como doença pré-existente hipertensão, já diabetes e depressão foram identificados em apenas $14,29 \%$ da amostra. Do total, 13 mulheres relataram não ter como rotina a prática de exercício físico e apenas uma possui o hábito de realizar exercícios físicos rotineiramente.

Para uma melhor identificação do nível da fadiga no decorrer do tratamento quimioterápico, cada mulher foi avaliada semanalmente: no dia da aplicação da quimioterapia ambulatorial (D1), no oitavo dia (D8) e no décimo quinto dia (D15) após a administração das medicações. Desta forma, a avaliação presencial, no "D1", foi realizada em todas, num total de 
Pictograma de fadiga durante o uso de quimioterápico no tratamento do câncer de mama $\mid 8$

56 avaliações devido à obrigatoriedade da presença destas para a aplicação da quimioterapia ambulatorial.

No entanto, as avaliações “D8” e “D15” não atingiram a totalidade, pois eram realizadas por meio de contato telefônico e por vezes, não eram encontradas em suas residências ou não atendiam ao telefone celular. Por isso, em relação à primeira etapa do tratamento que consiste na administração das drogas doxorrubicina + ciclofosfamida, o total de avaliações no oitavo dia (D8) foi 51 e no décimo quinto dia (D15) apenas 47.

Em relação à fase da aplicação do docetaxel, foram obtidas 49 avaliações no momento D8 e 42 avaliações no momento D15. Logo, no decorrer da pesquisa foram realizadas $301(89,58 \%)$ avaliações e apenas 35 (10,42\%) avaliações foram perdidas devido aos fatores supracitados. Os percentuais apresentados a seguir neste trabalho tomaram como totalidade (100\%) o número de avaliações realizadas que variaram em razão das dificuldades citadas.

O presente estudo é um dos poucos a acompanhar semanalmente o nível da fadiga em mulheres com câncer de mama ao longo de todo o tratamento quimioterápico. O protocolo terapêutico é dividido em duas fases, a primeira consiste em quatro sessões de doxorrubicina + ciclofosfamida seguido por mais quatro aplicações de docetaxel, logo, essas medicações são administradas em momentos distintos e apresentam efeitos colaterais diferentes. Desta forma, a avaliação da fadiga foi realizada em cada etapa do tratamento e foram divididas em quatro gráficos para uma melhor compreensão e dimensionamento do sintoma.

$\mathrm{Na}$ primeira etapa do tratamento, $44,64 \%(\mathrm{n}=25)$ participantes se recuperaram totalmente no dia da aplicação da quimioterapia ambulatorial (D1) ou já iniciaram o protocolo sem fadiga, pois informaram que não estavam se sentindo cansadas, outras $44,64 \%$ ( $\mathrm{n}=25)$ mulheres apresentaram um cansaço leve, $8,93 \%(\mathrm{n}=5)$ cansaço moderado e somente $1,79 \%$ ( $\mathrm{n}=1$ ) muito cansada. Durante a primeira semana (D8), 29,41\% (n =15) participantes não relataram cansaço, $31,37 \%(n=16)$ leve cansaço, 25,49\% ( $n=13)$ cansaço moderado, $11,77 \%(n=6)$ muito cansaço e 
9 | Vaz DC, Silva VRF, Silva RCL, Santiago LC, Silva CRL

apenas $1,96 \%(n=1)$ extremamente cansada. Já no décimo quinto dia (D15), o sintoma foi pouco relatado na maioria dos indivíduos, uma vez que, 40,42\% (n=19) já haviam se restabelecido, 42,55\% ( $\mathrm{n}=20)$ notaram um leve cansaço, $14,9 \%(\mathrm{n}=7)$ cansaço moderado e apenas $2,13 \%(\mathrm{n}=1)$ cansaço extremo.

Em relação à interferência do cansaço na realização de tarefas rotineiras, no dia do tratamento ambulatorial, 46,43\% $(\mathrm{n}=26)$ das participantes não relataram limitações em seu cotidiano, $42,86 \%(\mathrm{n}=24)$ relataram que podiam fazer quase tudo que compõe sua rotina, $7,14 \%$ (n = 4) mulheres relataram limitações para algumas coisas e outras 3,57\% (n=2) avaliaram que só fariam as coisas habituais que fossem realmente necessárias. Durante a primeira semana (D8), o número de pacientes que não foram afetadas em sua rotina diminuiu $29,41 \%$ ( $n=15)$ conseguiam fazer tudo e 33,33\% (n=17) quase tudo e, por conseguinte, o número de pacientes com limitações cotidianas aumentou, sendo $12(23,53 \%)$ as pacientes que ainda conseguiam obedecer a algumas das rotinas e $6(11,77 \%)$ as que faziam apenas o indispensável. Apenas 1 (1,96\%) ficou impedida de desempenhar qualquer tarefa da sua rotina.

$\mathrm{Na}$ segunda semana (D15), ainda em relação à rotina, houve recuperação de parcela considerável das pacientes, aumentando o número de indivíduos que não se sentiam afetados pela fadiga em sua rotina, $19(40,42 \%)$ e $21(44,68 \%)$ conseguiam fazer tudo ou quase tudo respectivamente e os indicadores mais graves foram menos frequentes dentre as respostas sendo $6(12,77 \%)$ as pacientes que faziam algumas das tarefas e novamente apenas $1(2,13 \%)$ paciente conseguiu desempenhar muito pouco as rotinas. As Figuras 2 e 3 descrevem a avaliação do nível da fadiga durante a etapa da administração das medicações doxorrubicina + ciclofosfamida. 
Pictograma de fadiga durante o uso de quimioterápico no tratamento do câncer de mama | 10

Figuras 2 e 3 - Acompanhamento da fadiga em mulheres em tratamento quimioterápico com doxorrubicina + ciclofosfamida utilizando o instrumento do Pictograma da Fadiga.
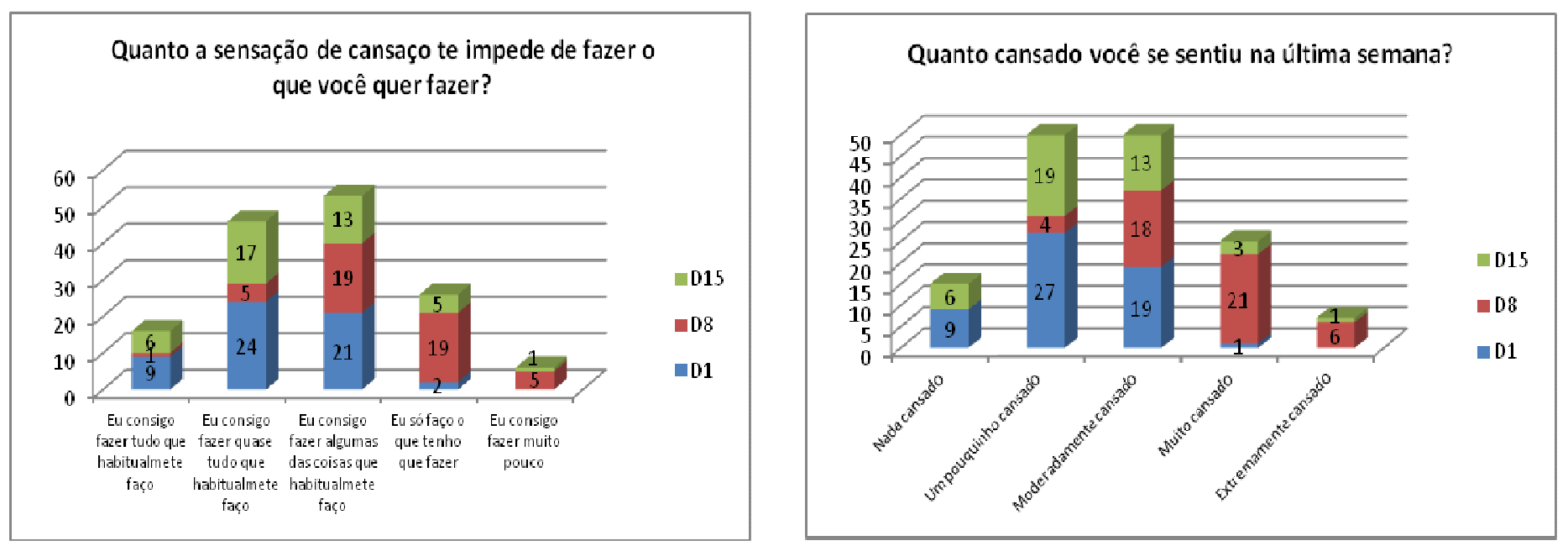

Fonte: dados da pesquisa, Rio de Janeiro - 2017.

A segunda fase do tratamento, que inclui as aplicações de docetaxel, o estudo demonstrou que o nível de fadiga aumentou significativamente em relação à etapa anterior e que houve um impacto na diminuição da realização das atividades diárias. A maior parte das pacientes 48,21\% ( $\mathrm{n}=27$ ); avaliou o nível da fadiga como leve no dia do tratamento (D1), 33,93\% ( $\mathrm{n}=19$ ) como moderadamente cansada, $1,79 \%(\mathrm{n}=1)$ como muito cansada, sendo assim, não se recuperaram plenamente em relação aos ciclos anteriores. Apenas $16,07 \%(\mathrm{n}=9)$ mulheres declaram estar completamente restabelecidas do sintoma no D1.

Na semana seguinte (D8) a intensidade da fadiga aumentou abruptamente, apresentando uma preponderância entre "muito cansado" 42,86\% (n = 21) e "moderadamente cansada" 36,74\% $(\mathrm{n}=18), 8,16 \%(\mathrm{n}=4)$ indivíduos referiram um cansaço leve e $12,24 \%(\mathrm{n}=6)$ como extremamente cansadas. Já no décimo quinto dia (D15) após a administração da medicação houve uma redução do sintoma na maioria das participantes, pois $14,29 \%(n=6)$ pacientes estavam revigoradas, $45,24 \%$ ( $n=19$ ) referiram estar "um pouquinho cansada”, 30,95\% ( $\mathrm{n}=13)$ moderadamente cansadas, $7,14 \%(n=3)$ muito cansadas e somente $2,38 \%(n=1)$ extremamente cansada. 
11 | Vaz DC, Silva VRF, Silva RCL, Santiago LC, Silva CRL

Em relação às atividades diárias, no dia do tratamento (D1) 16,07\% (n=9) pacientes informaram que estavam executando normalmente todas suas tarefas, $42,86 \%$ ( $n=24$ ) faziam quase tudo, 37,5\% ( $n=21)$ algumas coisas e 3,57\% $(n=2)$ somente o fundamental. Na semana seguinte (D8), houve uma limitação expressiva no desempenho das atividades cotidianas, 10,20\% $(\mathrm{n}=5)$ mulheres referiram fazer quase todas as tarefas, $38,78 \%(\mathrm{n}=19)$ somente algumas coisas, $38,78 \%(n=19)$ apenas o relevante, $10,20 \%(n=5)$ ficaram impossibilitadas de executar qualquer atribuição e exclusivamente $2,04 \%(n=1)$ manifestou nenhuma incapacidade para praticar seus afazeres.

No décimo quinto dia (D15), ocorreu o restabelecimento significativo no cumprimento das tarefas habituais, $40,48 \%(n=17)$ estavam realizando quase todas as suas atividades; $14,29 \%$ ( $\mathrm{n}=6)$ não manifestaram nenhum impedimento no desempenho das tarefas; 30,95\% ( $\mathrm{n}=13$ ) exerceram quase tudo, $11,90 \%(n=5)$ o imprescindível e apenas $2,38 \%(n=1)$ ficou impossibilitada para executar qualquer atividade. As Figuras 4 e 5 descrevem a avaliação do nível da fadiga durante a etapa da administração da medicação docetaxel.

Figuras 4 e 5 - Acompanhamento da fadiga em mulheres em tratamento quimioterápico com docetaxel utilizando o instrumento do Pictograma da Fadiga.
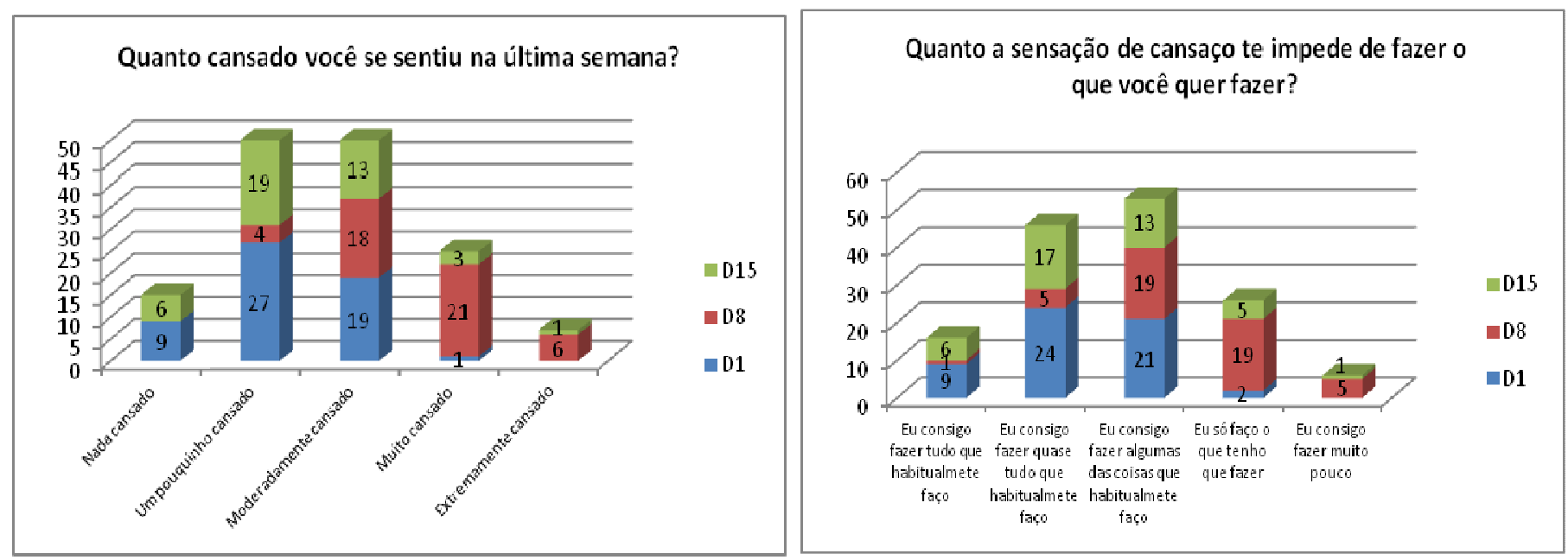

Fonte: dados da pesquisa, Rio de Janeiro - 2017. 
Pictograma de fadiga durante o uso de quimioterápico no tratamento do câncer de mama | 12

\section{Discussão}

Apesar das evidências científicas, relatarem que a quimioterapia tem como consequência a oscilação da fadiga ao longo do tratamento quimioterápico, estudos avaliaram diariamente ou semanalmente a presença deste sintoma, ${ }^{2-3}$ eles avaliaram o sintoma periodicamente, normalmente, no dia da infusão da quimioterapia. Porém, a investigação levando em consideração somente o dia do tratamento não demonstra completamente a experiência após a infusão das medicações no domicílio, pois o pico da fadiga não foi identificado e geralmente, os pacientes recebem o tratamento no dia que apresentam o menor nível da fadiga, uma vez que, precisam estar restabelecidos para a aplicação do próximo ciclo..$^{5,7}$

Esse sintoma tem impacto negativo no trabalho, nas relações sociais, no humor e nas atividades diárias, além de causar prejuízo significativo na qualidade de vida geral durante e após o tratamento, sendo considerado um preditor de menores taxas de sobrevida. Alguns estudos em pacientes com câncer de mama submetidas ao tratamento quimioterápico sugerem que a fadiga tem um padrão semelhante à “montanha-russa”, pois os níveis são mais elevados durante a primeira semana após a infusão da quimioterapia e em seguida começa a reduzir antes da próxima aplicação. ${ }^{6-8}$ No presente estudo ficou evidente que a fadiga ocorre ao longo das duas etapas do tratamento quimioterápico. Porém, na primeira etapa o sintoma ocorreu em menor gravidade e a maioria das pacientes conseguiu se restabelecer até o dia da próxima infusão de quimioterapia, não atingindo elevada interferência no desempenho das atividades diárias das pacientes. Já em relação à segunda etapa do protocolo, com a infusão do medicamento docetaxel, este sintoma foi mais acentuado e recorrente durante todo o tratamento, apresentando menores índices de recuperação para o dia da aplicação do próximo ciclo quimioterápico.

Tamanho é o impacto para os cuidados de enfermagem, a fadiga foi incluída como variável em vários estudos devido à sua alta prevalência e impacto na qualidade de vida dessas 
13 | Vaz DC, Silva VRF, Silva RCL, Santiago LC, Silva CRL

mulheres. Dada sua complexidade, está contemplada na classificação dos diagnósticos de enfermagem (DE) da Nanda International (NANDA-I), que descreve muitas características definidoras $(C D)$ que devem estar presentes para o diagnóstico ser estabelecido. O ND Fatigue apresenta dezesseis DCs, a maioria com subjetividade dos sintomas, fator que pode dificultar sua identificação. Enfatizamos que a identificação adequada de um DE é de suma importância para orientar as intervenções médicas. ${ }^{9-12}$

A avaliação D8 apresentou números mais elevados do sintoma em quantidade e em gravidade, tendo forte impacto sobre a execução de atividades diárias. Logo, o acompanhamento no decorrer do tratamento evidenciou que a fadiga foi progressiva e teve repercussão significativa na realização das atividades habituais. Um estudo ${ }^{7}$ com 130 mulheres com câncer de mama mostrou que a prevalência do diagnóstico de enfermagem para fadiga foi estimada em 21,9\% pelo modelo de classe latente. Essas mulheres tiveram sua capacidade comprometida em manter o nível habitual de atividade física. Esse resultado demostra que esse indicador pode ser a melhor medida determinar os estágios iniciais da fadiga, tal como diagnóstico de enfermagem.

O estudo ${ }^{8}$ ainda concluiu que a fadiga diminuiu a execução das atividades rotineiras, assim como, mulheres submetidas à mastectomia apresentaram níveis de fadiga maiores independente do tratamento quimioterápico adotado em relação àquelas que passaram por segmentectomia. Outro dado relevante foi que pacientes submetidas a um maior número de sessões de radioterapia e intervalos menores entre a realização da última aplicação da radioterapia e a mensuração do sintoma relataram maior nível de fadiga. ${ }^{8,13}$

A detecção precoce deste sintoma, usando escalas adequadas, é relevante para propor tratamentos adequados e alcançar melhores condições clínicas, adesão e continuidade do tratamento. Porém, o profissional de saúde teve ter o conhecimento que raramente a fadiga surge como sintoma isolado, está frequentemente associado a outros sintomas tais como: dor, depressão, alterações de sono, anemia e caquexia, entre outros, constituindo assim um 
Pictograma de fadiga durante o uso de quimioterápico no tratamento do câncer de mama $\mid 14$

agrupamento de sintomas. $^{9}$ Sendo assim, a investigação e o tratamento dos sintomas relacionados devem fazer parte da conduta terapêutica da fadiga.

Este sintoma pode ter um impacto extremamente negativo na qualidade de vida e atividades diárias dos pacientes, podendo interferir na adesão ao tratamento e, consequentemente, afetar o cumprimento do regime terapêutico proposto ou até mesmo causar a sua interrupção prematura. ${ }^{10} \mathrm{O}$ uso de escalas adequadas é de suma importância na detecção da fadiga enquanto sintoma e na proposição de tratamentos adequados. A adesão e a continuidade do tratamento dependem diretamente de que os pacientes atinjam o melhor quadro clínico possível, que por sua vez depende de um acompanhamento eficaz pelo profissional de saúde. Tal eficácia passa, inclusive, pela necessária sensibilidade por parte do profissional de saúde de que a fadiga raramente surge como sintoma isolado, sendo decorrente ou podendo decorrer de outro sintoma ou do agrupamento de vários deles. Assim, a conduta terapêutica da fadiga requer que o acompanhamento inclua a percepção e a investigação de outros sintomas..$^{8,10,13}$

\section{Conclusão}

As mulheres participantes deste estudo possuíam idade média de 57,3 anos, portanto, um grupo ainda joven, sendo a metade delas com nível médio de instrução. Somente cinco estavam empregadas e a maioria, aposentada, provavelmente, em função da doença. A fadiga ocorreu ao longo das duas primeiras etapas do tratamento, mas com a utilização docetaxel, este sintoma foi mais acentuado e recorrente durante todo o tratamento. Assim, atividades diárias como caminhar, se exercitar, limpar a casa, cozinhar e até comer podem se tornar grandes desafios. As limitações do câncer e seu tratamento também estão associadas a uma perda progressiva de massa muscular, reduzindo a força muscular e a capacidade do paciente de executar tarefas simples. 
15 | Vaz DC, Silva VRF, Silva RCL, Santiago LC, Silva CRL

A utilização do Pictograma da Fadiga pode contribuir de maneira significativa no cuidar em enfermagem na medida em que se apresenta como um importante instrumento de acompanhamento, simples e com baixo custo. Some-se ainda uma série de vantagens proporcionadas pela adoção da escala, entre elas, avaliação, acompanhamento e padronização do registro, o aperfeiçoamento das condutas prestadas e, principalmente, a criação de indicadores da assistência de enfermagem aos pacientes com quadro de fadiga, algo de grande utilidade considerando-se as altas taxas de incidência deste sintoma dentre as mulheres em tratamento quimioterápico.

Este instrumento demonstrou ser de fácil compreensão e rápida aplicação. Sua melhor utilização é verificada nos protocolos com intervalo de 21 dias com avaliações semanais do paciente, uma vez que, se for perguntado ao paciente no dia do tratamento "quanto cansado você se sentiu na última semana” não será identificado o período mais crítico do sintoma, pois já estará no período de recuperação.

A partir deste estudo, acredita-se na relevância de se conhecer e aplicar instrumentos de acompanhamento da população atendida, com o propósito de minimizar custos e aumentar o conforto dessas mulheres, estimulando sua participação ativa no tratamento e menos sofrimento no processo reabilitação. Além disso, tem-se a intenção de estimular a produção de pesquisas que possam relacionar a utilização do Pictograma de fadiga nas mais diversas situações de cuidado. Como principal limitação, admite-se a possibilidade de viés das respostas obtidas por telefone, bem como a impossibilidade de uma análise estatística mais refinada em virtude do tamanho da amostra.

\section{Referências}

1. Instituto Nacional de Câncer (BR). Estimativa 2016: incidência de câncer no Brasil. Rio de Janeiro (RJ): Inca; 2015. 
Pictograma de fadiga durante o uso de quimioterápico no tratamento do câncer de mama | 16

2. Castanhel FD, Liberali R. Redução do estresse baseado na atenção plena nos sintomas do câncer de mama: revisão sistemática e metanálise. Einstein [Internet]. 2018 [acesso em 2019 set 03];16(4):eRW4383. Disponível em: https://journal.einstein.br/pt-br/article/reducao-de-estresse-baseada-em-mindfulnessnos-sintomas-do-cancer-de-mama-revisao-sistematica-e-metanalise/

doi: 10.31744/einstein_journal/2018RW4383

3. Rautalin M, Färkkilä N, Sintonen H, Saarto T, Jahkola T, Roine RP, et al. Health-related quality of life in different states of breast cancer-comparing different instruments. Acta Oncol [Internet]. 2017 [acesso em 2018 abr 30];57(5):622-8. Disponível em: https://www.tandfonline.com/doi/abs/10.1080/0284186X.2017.1400683?journalCode=ionc20\# https://doi.org/10.1080/0284186X.2017.1400683

4. Mourão CML, Fernandes AFC, Moreira DP, Martins MC. Entrevista motivacional no suporte social de cuidadores de pacientes com câncer de mama em quimioterapia. Rev Esc Enferm USP [Internet]. 2017 [acesso em 2018 abr 30]; 51:e03268. Disponível em: http://www.scielo.br/scielo.php?pid=S008062342017000100471\&script=sci_abstract\&tlng=pt. Epub Dec 18, 2017. doi: http://dx.doi.org/10.1590/s1980220x2017001803268

5. Mota CF, Pimenta CAM, Fitch MI. Pictograma de fadiga: uma alternativa para avaliação da intensidade e impacto da fadiga. Rev Esc Enferm USP [Internet]. 2009 [acesso em 2018 abr 30];43 Esp:1080-7. Disponível em: http://www.scielo.br/scielo.php?pid=S008062342009000500012\&script=sci_abstract\&tlng=pt doi: http://dx.doi.org/10.1590/S0080-62342009000500012

6. Balsanelli ACS, Grossi SAA. Fatores preditores da esperança entre mulheres com câncer de mama durante o tratamento quimioterápico. Rev Esc Enferm USP [Internet]. 2016 [acesso em 2018 abr 30];50(6):898-904. Disponível em: http://www.scielo.br/pdf/reeusp/v50n6/pt_0080-6234-reeusp-50-0600898.pdf doi: http://dx.doi.org/10.1590/S0080-623420160000700004

7. Rocha SR, Santos MCL, Lopes MVO, Rodrigues AB, Sousa VEC, Aquino CQ, et al. Acurácia das características definidoras do diagnóstico de enfermagem para fadiga em mulheres sob radioterapia. Rev Bras Enferm [Internet]. 2018 [acesso em 2019 set 03];71(Supl 3):1445-52. Disponível em: http://www.scielo.br/pdf/reben/v71s3/pt_0034-7167-reben-71-s3-1445.pdf doi: http://dx.doi.org/10.1590/0034-7167-2017-0549

8. Pakzad R, Mohammadian-Hafshejani A, Khosravi B, Soltani S, Pakzad I, Mohammadian M, et al. The incidence and mortality of esophageal cancer and their relationship to development in Asia. Ann Transl Med [Internet]. 2016 [acesso em 2018 abr 30];4(2):29. Disponível em: https://www.ncbi.nlm.nih.gov/pmc/articles/PMC4731602/

9. Vieira RA, Lourenço TS, Mauad EC, Moreira Filho VG, Peres SV, Silva TB, et al. Barriers related to non-adherence in a mammography breast-screening program during the implementation period in the interior of São Paulo State, Brazil. J Epidemiol Glob Health [Internet]. 2015 [acesso em 2018 abr 30];5(3):211-9. Disponível em: https://www.ncbi.nlm.nih.gov/pubmed/26231397 
10. Kokkonen K, Saarto T, Mäkinen T, Pohjola L, Kautio H, Järvenpää S, et al. The functional capacity and quality of life of women with advanced breast cancer. Breast Cancer [Internet]. 2017 [acesso em 2018 mai 08]; 24(1):128-36. Disponível em: https://www.ncbi.nlm.nih.gov/pubmed/27002988 doi: $10.1007 / \mathrm{s} 12282-016-0687-2$

11. Bower JE. Fadiga relacionada ao câncer: mecanismos, fatores de risco e tratamentos. Nat Rev Clin Oncol [Internet]. 2014 [acesso em 2017 maio 27];11(10): 597-609. Disponível em: https:/www.ncbi.nlm.nih.gov/pmc/articles/PMC4664449/pdf/nihms664147.pdf

12. Curt GA, Breitbart W, Cella D, Groopman JE, Horning SJ, Itri LM, et al. Impacto da fadiga relacionada ao câncer na vida de pacientes: novas descobertas da coalizão de fadiga. Oncologist [Internet]. 2000 [acesso em 2017 jun 27];5:353-60. Disponível em: http://theoncologist.alphamedpress.org/content/5/5/353.full.pdf+html

13. Lamino DA, Pimenta CAM, Braga PE, Mota DDCF. Fadiga clinicamente relevante em mulheres com câncer de mama: prevalência e fatores associados. Investig Enferm Imagen Desarro [Internet]. 2015 [acesso em 2017 jun 27];17(1):65-76. Disponível em: http://www.redalyc.org/pdf/1452/Resumenes/Abstract_145233516005_2.pdf

\section{Autor correspondente}

Nome: Carlos Roberto Lyra da Silva

E-mail: profunirio@gmail.com

Endereço: EEAP, sala 601. Rua Dr. Xavier Sigaud, 290 - Urca, Rio de Janeiro - RJ. Brasil.

CEP: $22290-180$

\section{Contribuições de Autoria}

\section{1 - Daniele Copello Vaz}

Concepção e planejamento do projeto de pesquisa, obtenção, análise e interpretação dos dados, redação.

\section{2 - Viviane Reis Fontes da Silva}

Revisão crítica.

\section{3 - Roberto Carlos Lyra da Silva}

Revisão crítica.

\section{4 - Luiz Carlos Santiago}

Revisão crítica.

\section{5 - Carlos Roberto Lyra da Silva}

Análise e interpretação dos dados, redação, revisão crítica. 
Pictograma de fadiga durante o uso de quimioterápico no tratamento do câncer de mama | 18

\section{Como citar este artigo}

Vaz DC, Silva VRF, Silva RCL, Santiago LC, Silva CRL. Pictograma de fadiga durante o uso de quimioterápico no tratamento do câncer de mama. Rev. Enferm. UFSM. 2019 [Acesso em: Anos Mês Dia];vol.9: e53: P1-18. DOI:https://doi.org/10.5902/2179769232076 